EPJ Web of Conferences 109,04001 (2016)

DOI: 10.1051/epjconf/201610904001

(C) Owned by the authors, published by EDP Sciences, 2016

\title{
Reactions on the surface and inside of neutron stars
}

\author{
K. E. Rehm ${ }^{1 a}$ \\ ${ }^{1}$ Argonne National Laboratory, Physics Division, Argonne, IL 60439, USA
}

\begin{abstract}
Measurements from orbiting X-ray satellites during the last decades have provided us with a wealth of information about nuclear reactions thought to occur in the extreme, highdensity environment of neutron stars. With radioactive ion beams from first-generation facilities we have begun to study some of these processes in the laboratory. In this contribution I report on experiments performed with radioactive beams from the ATLAS accelerator at Argonne. I will discuss the nuclear physics of X-ray bursts and super-bursts, the production of in-flight radioactive beams, as well as novel detectors which are used in these experiments.
\end{abstract}

\section{Introduction}

X-ray bursts are the most frequent stellar explosions in the Universe. First observed in 1975 [1] they were quickly explained as thermonuclear runaways occurring in low-mass X-ray binary systems where hydrogen/helium from an orbiting main sequence or red-giant star is being transferred onto the surface of a neutrons star [2]. The strong gravitational forces in these systems have the material fall on the surface with energies of about $200 \mathrm{MeV} / \mathrm{u}$ resulting in a continuous flux of X-rays. If enough material has been accumulated the hydrogen/helium mixture explodes with an energy release of $10^{38-39}$ ergs leading to a sudden increase in X-ray flux lasting from tens of seconds to several minutes. With more than a 100 sources found so far and repetition rates ranging from one to several hours, a large data base of these events has been accumulated [3]. In order to separate these 'standard' X-ray bursts from events exhibiting shorter recurrence times $(\sim 10 \mathrm{sec})$ a classification system into type I and type II X-ray bursts has been introduced with type I bursts being associated with thermonuclear runaways and type II with accretion instabilities. While X-ray bursts do not contribute to the chemical evolution of the universe they give us valuable information about nuclear processes occurring in very dense nuclear matter. The diversity in the time structures [4] and the occurrence of burst multiplets [5] are examples of the research areas being studied by different groups.

In addition to the type-I and II X-ray bursts mentioned above a few so-called superbursts have recently been observed [6]. Superbursts last several hours (compared to tens of seconds) and thus have an energy release of about $10^{42}$ ergs. These superbursts which have recurrence times of about a year are thought to occur deeper in the 'ocean' of a neutron star, possibly involving unstable carbon burning and other reactions between neutron-rich nuclei. In this contribution I will discuss some of the nuclear physics which is associated with type I X-ray bursts and with superbursts.

${ }^{\text {a }}$ Corresponding author: rehm@anl.gov

This is an Open Access article distributed under the terms of the Creative Commons Attribution License 4.0, which permits unrestricted use, distribution, and reproduction in any medium, provided the original work is properly cited. 


\section{Nuclear reactions in type-I X-ray bursts}

\subsection{Reactions on the surface of neutron stars}

The short time frames involved in explosive nucleosynthesis requires a reaction path which passes through nuclei with very short half-lives and, thus, is located away from the valley of maximum $\beta$ stability. Due to the hydrogen-rich environment on the surface of an accreting neutron star, this path proceeds through nuclei on the proton-rich side of the mass valley close to the proton drip line. A typical reaction path is shown in Fig. 1 [7]. For a quantitative understanding of this reaction path one needs a robust knowledge of the underlying nuclear properties: Masses and binding energies of $\beta$ unstable nuclei close to the drip line, half-lives for $\beta$ decays and the associated nuclear-reaction rates

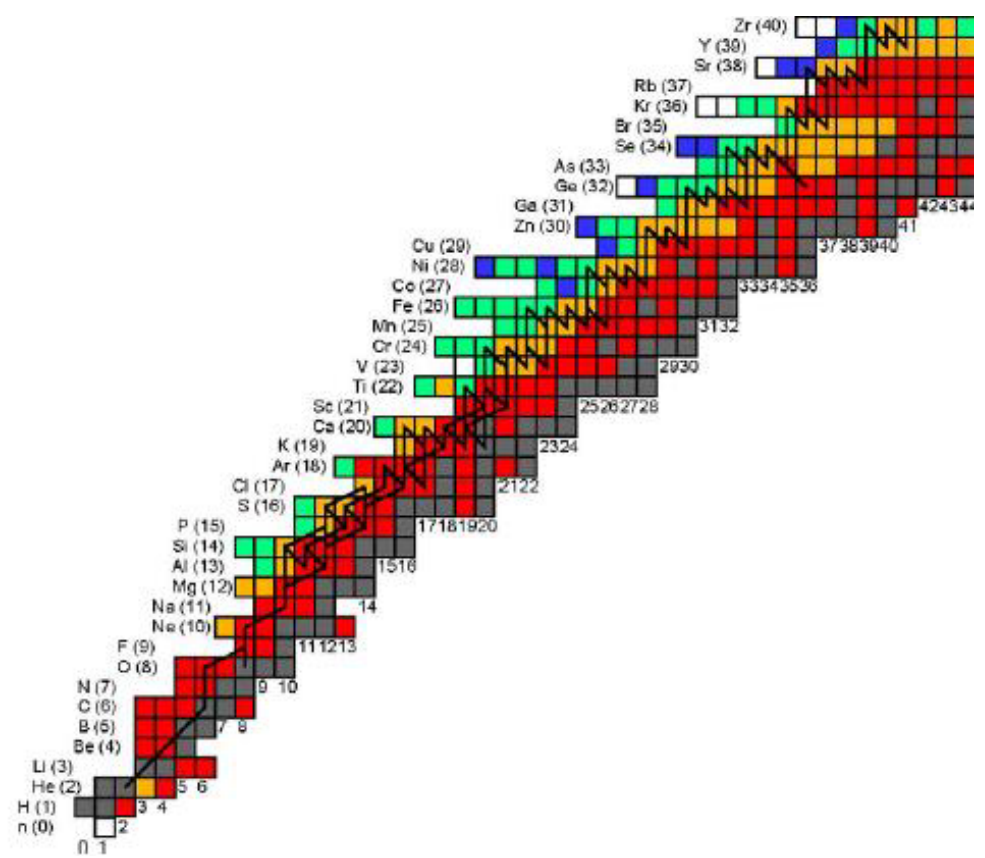

Figure 1: Reaction path in the rapid-proton (rp) process taken from Ref. [7]. Stable nuclei are shown in grey, nuclides with mass known to better than $10 \mathrm{keV}$ are red and other nuclei with experimentally known masses are orange. Nuclides with experimentally know half-lives, but unknown masses are green, and nuclei that have only been identified in nuclear reactions are blue.

for $(\mathrm{p}, \gamma)$ and $(\alpha, \mathrm{p})$ reactions. During the past decade, measurements at several laboratories worldwide have given us a good understanding of the masses as well the $\beta$ decay rates in this region of the nuclear chart [8]. In this contribution I will concentrate on measurements of the important nuclear reaction rates.

As can be seen from Fig. 1 the reaction path for heavier nuclei is dominated by $(\mathrm{p}, \gamma)$ reactions on unstable nuclei followed by $\beta$ decays. For lighter nuclei $(\mathrm{A}<40)$ the lower Coulomb barrier allows for the competition from the so-called $(\alpha, p)$ process, where in one reaction step three particles are transferred, which is equivalent to three $(\mathrm{p}, \gamma)$ reactions and two $\beta$ decays.

While in the astrophysical environment these reactions occur naturally in the hot stellar hydrogen/helium plasma, measurements in terrestrial laboratories have to be studied individually in inverse kinematics with beams of short-lived isotopes bombarding hydrogen or helium targets. 
Although these beams are available at a variety of radioactive beam facilities, only a few $(\mathrm{p}, \gamma)$ and $(\alpha, p)$ measurements have been performed so far [7].

\subsection{Reactions in the ocean of a neutron star}

While studies of type-I X-ray bursts have given us some information about the nuclear reactions occurring in the atmosphere of a neutron star our knowledge of the underlying nuclear physics of superbursts is much more rudimentary. While we know that they are powered by nuclear reactions involving the ashes of type-I X-ray bursts, which moved from the atmosphere into the so-called ocean of a neutron star, the actual composition of the ashes inside a neutron star is not so well known.

\section{Studies at the ATLAS accelerator}

At the heavy ion accelerator ATLAS at Argonne National Laboratory we have begun to study some of the reactions critical to our understanding of type I X-ray bursts and superbursts using beams on the proton-rich as well as on the neutron-rich side of the mass valley. The beams were produced via the in-flight technique [9] by bombarding a hydrogen- or deuterium-filled production target with heaviermass stable ion beams. The radioactive beams are produced in the gas cell through reactions in inverse kinematics such as ${ }^{1} \mathrm{H}\left({ }^{10} \mathrm{~B},{ }^{10} \mathrm{C}\right) \mathrm{n},{ }^{2} \mathrm{H}\left({ }^{32} \mathrm{~S},{ }^{33} \mathrm{Cl}\right) \mathrm{n}$, or ${ }^{2} \mathrm{H}\left({ }^{14} \mathrm{C},{ }^{15} \mathrm{C}\right) \mathrm{p}$. These secondary beams are then separated from the primary beam by a $26^{\circ}$ bending magnet and a RF-sweeper [10] located after the magnet. With these radioactive beams, nuclear reactions, which are relevant to the $(\alpha, p)$ process in type-I x-ray bursts and fusion reactions thought to be of importance in superbursts, have been investigated. Some of these reactions will be discussed in more detail below.

\subsection{Reactions in the $(\alpha, p)$ process}

As can be seen from the reaction path in Fig. 1 the $(\alpha, p)$ reactions play a role mainly in the mass range $\mathrm{A}<40$, providing a 'shortcut' in the reaction flow by avoiding a large number of sometimes slow $\beta$ decays. Starting from ${ }^{14} \mathrm{O}$ the $(\alpha, \mathrm{p})$ process proceeds via the sequence:

$$
{ }^{14} \mathrm{O}(\alpha, \mathrm{p}){ }^{17} \mathrm{~F}(\mathrm{p}, \gamma){ }^{18} \mathrm{Ne}(\alpha, \mathrm{p}){ }^{21} \mathrm{Na}(\mathrm{p}, \gamma){ }^{22} \mathrm{Mg}(\alpha, \mathrm{p}){ }^{25} \mathrm{Al}(\mathrm{p}, \gamma){ }^{26} \mathrm{Si}(\alpha, \mathrm{p})^{29} \mathrm{P}(\mathrm{p}, \gamma){ }^{30} \mathrm{~S}(\alpha, \mathrm{p})^{33} \mathrm{Cl}(\mathrm{p}, \gamma)^{34} \mathrm{Ar}(\alpha, \mathrm{p})^{37} \mathrm{~K}(\mathrm{p}, \gamma){ }^{38} \mathrm{Ca}
$$

For experimental studies of these reactions it is easier to produce beams of ${ }^{14} \mathrm{O},{ }^{17} \mathrm{~F},{ }^{21} \mathrm{Na},{ }^{25} \mathrm{Al},{ }^{29} \mathrm{P}$, ${ }^{33} \mathrm{Cl}$ and ${ }^{37} \mathrm{~K}$, via inverse $(\mathrm{p}, \mathrm{n})$ or $(\mathrm{d}, \mathrm{n})$ reactions, when compared to beams of ${ }^{18} \mathrm{Ne},{ }^{22} \mathrm{Mg},{ }^{26} \mathrm{Si},{ }^{30} \mathrm{~S}$, ${ }^{34} \mathrm{Ar}$ which require two-particle transfer reactions, such as $\left({ }^{3} \mathrm{He}, \mathrm{n}\right)$ or $\left({ }^{12} \mathrm{C},{ }^{10} \mathrm{Be}\right)$. For that reason many of the relevant $(\alpha, p)$ reactions mentioned above have been studied via the time-inverse $(p, \alpha)$ reaction using radioactive beams that are closer to the valley of stability. This technique, however, only gives information on the ground state to ground state reaction strength while the influence of excited states needs to be obtained using statistical model calculations. In several experiments performed during the past few years we have measured the cross sections of the time-inversed gs to gs yields for the ${ }^{22} \mathrm{Mg}(\alpha, \mathrm{p}){ }^{25} \mathrm{Al},{ }^{26} \mathrm{Si}(\alpha, \mathrm{p}){ }^{29} \mathrm{P},{ }^{30} \mathrm{~S}(\alpha, \mathrm{p}){ }^{33} \mathrm{Cl},{ }^{34} \mathrm{Ar}(\alpha, \mathrm{p}){ }^{37} \mathrm{~K}$ and ${ }^{30} \mathrm{P}(\alpha, \mathrm{p}){ }^{33} \mathrm{~S}$ [11-13] reactions. The first four systems require radioactive ion beams while the last reaction was performed with a stable ${ }^{33} \mathrm{~S}$ beam as a test case to study the experimental technique.

The experimental setup is shown in Fig. 2 [12]. The beams $\left({ }^{25} \mathrm{Al},{ }^{29} \mathrm{P},{ }^{33} \mathrm{~S},{ }^{33} \mathrm{Cl}\right.$ and $\left.{ }^{37} \mathrm{~K}\right)$ from the ATLAS accelerator were bombarding $\mathrm{CH}_{2}$ targets mounted in the scattering chamber of an Enge Split-Pole spectrograph. The $\alpha$ particles from the $(\mathrm{p}, \alpha)$ reaction were detected by a double-sided 
annular position sensitive Si detector covering the angular range of $\theta_{\mathrm{lab}}=8^{\circ}-25^{\circ}$. The corresponding heavy reaction products ${ }^{22} \mathrm{Mg},{ }^{26} \mathrm{Si},{ }^{30} \mathrm{P},{ }^{30} \mathrm{~S},{ }^{34} \mathrm{Ar}$ emitted in the angle range $\theta=1.5^{\circ}-3^{\circ}$ entered the splitpole spectrograph. To increase the detection efficiency of the system the magnet was operating in gas-

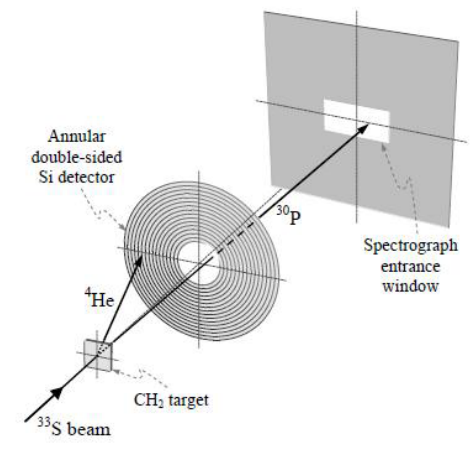

Figure 2: Schematic of the experimental setup used for measurements of the $(p, \alpha)$ reactions. See text for details.

filled mode [14]. Under these conditions charge-changing collision between the incident ions with nuclear charge $\mathrm{Z}$ and the nitrogen gas molecules in the magnetic field lead to an average charge state of the ions $\langle q\rangle$ which in first order is proportional to the average ion velocity $\langle\mathrm{v}\rangle\left(\langle\mathrm{q}\rangle \sim\langle\mathrm{v}\rangle \mathrm{Z}^{\gamma}\right.$ with an exponent $\gamma \sim 1 / 3)$. As a result the magnetic rigidity is in first order independent of the velocity resulting in a separation of the ions in the focal plane of the spectrometer according to their value $\mathrm{m} / \mathrm{Z}^{\gamma}$. This technique was first tested for the ${ }^{33} \mathrm{~S}(\mathrm{p}, \alpha){ }^{30} \mathrm{P}$ reaction with stable ${ }^{33} \mathrm{~S}$ beams in the energy range of about 120-200 MeV. Details of these tests have been published elsewhere [12].

Fig. 3 shows the excitation functions of the ${ }^{33} \mathrm{~S}(\mathrm{p}, \alpha){ }^{30} \mathrm{P}$ and ${ }^{33} \mathrm{Cl}(\mathrm{p}, \alpha){ }^{30} \mathrm{~S}$ reactions in the c.m. range of 4-7 $\mathrm{MeV}[11,12]$. Due to the higher intensities $\left(10^{8}\right.$ particles $\left./ \mathrm{sec}\right)$ available with the stable ${ }^{33} \mathrm{~S}$ beam
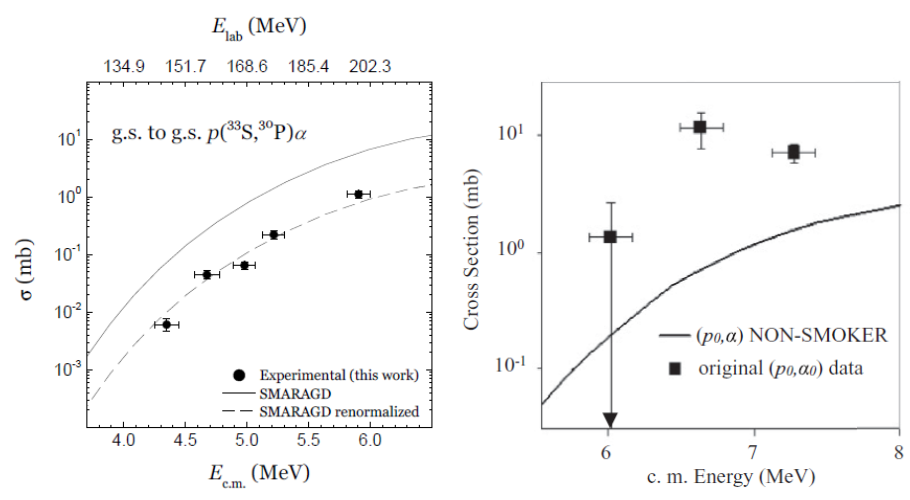

Figure 3: Excitation functions of the ${ }^{33} \mathrm{~S}(\mathrm{p}, \alpha){ }^{30} \mathrm{P}$ reaction (left) and ${ }^{33} \mathrm{Cl}(\mathrm{p}, \alpha){ }^{30} \mathrm{~S}$ reaction (right) measured with the gas-filled magnet technique. See Ref. 12 for more details.

the excitation function of the ${ }^{33} \mathrm{~S}(\mathrm{p}, \alpha)^{30} \mathrm{P}$ reaction could be measured down to the $5 \mu \mathrm{b}$ cross section region while the excitation function of the ${ }^{33} \mathrm{Cl}(\mathrm{p}, \alpha){ }^{30} \mathrm{~S}$ reaction measured with beam intensities of 
about $10^{5}$ particles/sec is so far limited to the $5-10 \mathrm{mb}$ reaction. The solid lines in Fig. 3 originate from statistical model calculations using the SMARAGD [15] code. As can be seen from Fig. 3 the experimental cross sections of the ${ }^{33} \mathrm{~S}(\mathrm{p}, \alpha){ }^{30} \mathrm{P}$ reaction are smaller than the statistical model predictions while the opposite is true for the ${ }^{33} \mathrm{Cl}(\mathrm{p}, \alpha)^{30} \mathrm{~S}$ reaction. It should be noted that the timeinverse of the ${ }^{33} \mathrm{Cl}(\mathrm{p}, \alpha){ }^{30} \mathrm{~S}$ reaction was predicted to cause a change in the shape of the X-ray burst in Ref. [16] with higher rates producing a two-peaked burst structure. In these simulations, however, the cross section was changed by a factor of 100 which is larger than what was experimentally observed. Results from the remaining $(\alpha, p)$ reactions are under analysis and preliminary results can be found in the literature [13].

\subsection{Fusion reactions in superbursts}

X-ray superbursts, as mentioned earlier, last several hours releasing 1000 times more energy than a normal burst with a recurrence time between bursts of about a year. These 'superbursts' are powered through nuclear reactions involving the ashes (e.g. carbon) produced in type-I bursts which have moved from the neutron star atmosphere deeper into the 'ocean'. It is estimated that ignition of carbon occurs at densities of $10^{9} \mathrm{~g} / \mathrm{cm}^{2}$, i.e. three orders of magnitude higher than that for regular bursts. Simulations of these processes, however, cannot reproduce the conditions that are needed in order to start carbon ignition. For that reason it has been argued that fusion cross sections involving neutronrich isotopes of carbon, oxygen and neon need to be included [17]. At the higher densities in the ocean of a neutron star the rising Fermi energy can induce electron capture reactions in the ashes of normal $\mathrm{X}$-ray bursts lowering their atomic number and producing very neutron-rich nuclei [18]. Finally in the vicinity of the neutron-star crust the nuclei can fuse via so-called pycno-nuclear reactions. In order to understand the origin of the superbursts a series of publications discussing fusion cross sections of neutron-rich carbon $\left({ }^{24} \mathrm{C}+{ }^{24} \mathrm{C}\right)$, oxygen $\left({ }^{24} \mathrm{O}+{ }^{24} \mathrm{O}\right)$ and neon $\left({ }^{40} \mathrm{Ne}+{ }^{40} \mathrm{Ne}\right)$ nuclei have recently been published $[17,19,20]$. A large fraction of the nuclei included in these calculations are outside the range of our present experimental capabilities $\left(\right.$ e.g. $\left.{ }^{24} \mathrm{C}+{ }^{24} \mathrm{C}\right)$. Below I describe a new measurement of the total fusion cross sections of ${ }^{12} \mathrm{C}+{ }^{10,12,13,14,15} \mathrm{C}$ using a new active target system.

Experiments with secondary ion beams at today's radioactive beam facilities suffer from low beam intensities. This complicates especially experiments where the energy dependence of the cross sections has to be measured, with fusion reactions being a prime example. For this reason no fusion experiments with radioactive beams have yet been performed at energies which are important to nuclear astrophysics. For these types of experiments we have developed a method that allows us to measure in one experiment a large fraction of an excitation function for fusion reactions between helium, carbon or neon and several radioactive ion beams at energies in the vicinity of the Coulomb barrier.

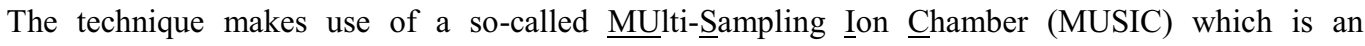
ionization chamber filled e.g. with $\mathrm{CH}_{4}$ gas which serves both as target and counting gas. Multisampling ionization chambers have been used in the past for experiments with relativistic heavy ions $[21,22]$ and a similar detector was employed for a measurement of the ${ }^{8} \operatorname{Li}(\alpha, n)^{11} \mathrm{~B}$ reaction $[23,24]$. Details about the technique used with this detector system are given in Ref. [25]. The anode of the detector is subdivided into 18 strips as shown in the lower part of Fig. 4. In measuring the ${ }^{12} \mathrm{C}+{ }^{15} \mathrm{C}$ reaction, the radioactive ${ }^{15} \mathrm{C}$ beam which enters the ion chamber through a $1.45 \mathrm{mg} / \mathrm{cm}^{2}$ Ti window and experiences an energy loss in the gas volume of each of the 18 anode strips corresponding to about $1.4 \mathrm{MeV}$ per strip at a $\mathrm{CH}_{4}$ pressure of 200 mbar. The signals from these beam-like events measured at the anode of the ion chamber are shown by the black lines in Fig. 4. With an incident ${ }^{15} \mathrm{C}$ beam energy of $49 \mathrm{MeV}$ the ${ }^{15} \mathrm{C}+{ }^{12} \mathrm{C}$ fusion reaction is sampled in the energy range $\mathrm{E}_{\mathrm{cm}}=9-18 \mathrm{MeV}$. A fusion reaction occurring in the gas volume of one of the anode strips (e.g. strip 4) is identified by beam-like energy losses in strip 1-3 followed by larger signals generated by the $\mathrm{C}+\mathrm{C}$ evaporation 
residues (ER) in the following 3-4 strips and no signal in the remaining strips as indicated by the red lines in Fig. 4

Since angular distributions of ERs from fusion reactions in this mass and energy range generally peak at $0^{\circ}$ and extend to scattering angles of about $35^{\circ}$ practically the full yield of the fusion reactions can be detected in the first 10-12 strips. Fusion events occurring in the last 4-6 strips will experience some losses in efficiency. Another advantage of the multi-sampling method is that the fusion measurement
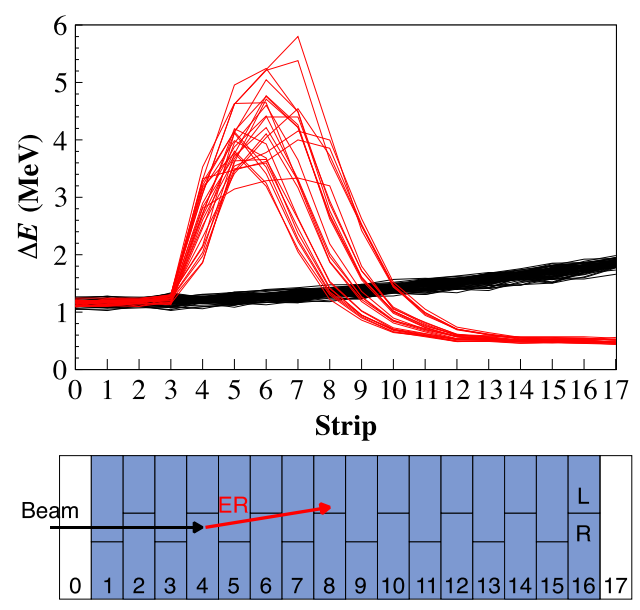

Figure 4: Experimental traces measured with the MUSIC detector. Black lines correspond to beam particles interacting with the gas target by ionization. Red lines are related to fusion events occurring in strip 4 as described in the text. They are characterized by a jump in $\Delta E$ followed by zero pulse height in strips higher than 12 .

is self-normalizing, since the beam and the fusion events at the various energies are obtained from the same detector using the same number of incident particles without the need to change the energy or to use a monitor detector. The fusion cross sections for ${ }^{10,12,13,14,15} \mathrm{C}$ on ${ }^{12} \mathrm{C}$ converted into astrophysical $\mathrm{S}$ factors are shown by the solid points in Fig. 5 [26]. The solid lines are calculations by Yakovlev et al. [20] which are based on tunneling through a barrier using the Sao Paulo potential. The red circles are $\mathrm{S}$ factors for the systems ${ }^{12,13} \mathrm{C}+{ }^{12} \mathrm{C}$ for which data in the energy range from about 3 to $30 \mathrm{MeV}$ are available [27,28]. Over the full energy range, theory and experiment agree to better than a factor of 2 . Also shown are the theoretical predictions for the system ${ }^{19} \mathrm{C}+{ }^{12} \mathrm{C}$ which is the heaviest carbon isotope that is predicted to be available at the next generation FRIB facility with beam intensities exceeding $10^{3}$ particles $/ \mathrm{sec}$.

A very good agreement of our data with the earlier measurements of Ref. $[27,28]$ for ${ }^{12,13} \mathrm{C}$ is observed. The energy dependence of the data is also well described by the predictions of Yakovlev et al. [20] especially in the energy range $\mathrm{E}_{\mathrm{cm}}<14 \mathrm{MeV}$, indicating that theoretical calculations should be able to make predictions which are accurate within a factor of about 2 . Similar measurements have 


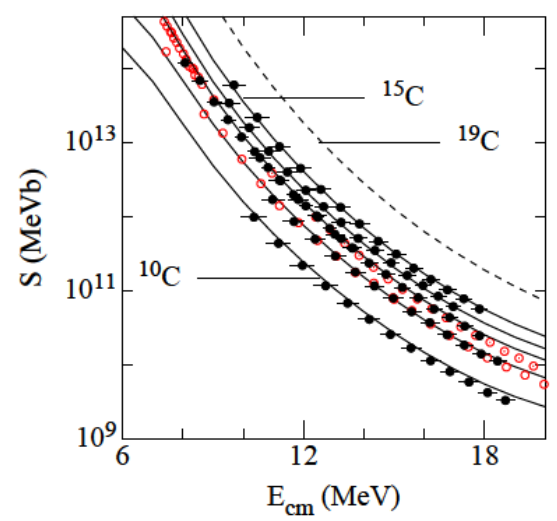

Figure 5: Solid points: Experimental data of the S-factors in the fusion reactions ${ }^{10,12,13,14,15} \mathrm{C}+{ }^{12} \mathrm{C}$ as measured with the MUSIC detector. Open circles: Experimental data for ${ }^{12,13} \mathrm{C}+{ }^{12} \mathrm{C}$ from Refs. [27,28]. Solid lines: theoretical $\mathrm{S}$ factors for the systems ${ }^{10,12,13,14,15} \mathrm{C}+{ }^{12} \mathrm{C}$ taken from the calculations of Yakovlev et al. [20]. The dashed line is the prediction for the system ${ }^{19} \mathrm{C}+{ }^{12} \mathrm{C}$.

been performed for the systems ${ }^{20} \mathrm{Ne}+{ }^{20} \mathrm{Ne}$ and ${ }^{22} \mathrm{Ne}+{ }^{22} \mathrm{Ne}$ [29], and experiments with radioactive $\mathrm{Ne}$ isotopes are planned for the future.

\subsection{Study of $(\alpha, p)$ and $(\alpha, n)$ reactions with MUSIC}

The ability of MUSIC to study fusion reactions involving low-intensity radioactive beams opens the possibility to investigate other reactions with this detector system as well. Earlier experiments [23] involving a larger detector system have been done for the system ${ }^{8} \mathrm{Li}(\alpha, n){ }^{11} \mathrm{~B}$. We have started to investigate whether MUSIC can separate $(\alpha, n)$ and $(\alpha, p)$ reactions for the systems ${ }^{17} \mathrm{O}(\alpha, n){ }^{20} \mathrm{Ne}$ and ${ }^{23} \mathrm{Na}(\alpha, \mathrm{p}){ }^{26} \mathrm{Mg},{ }^{23} \mathrm{Na}(\alpha, \mathrm{n}){ }^{26} \mathrm{Al}$, where data using other detection techniques can be found in the literature. For that we have filled MUSIC with helium gas at pressures of 200-400 Torr and bombarded the detector with incident beams of ${ }^{17} \mathrm{O}$ and ${ }^{23} \mathrm{Na}$ with intensities of about $5 \times 10^{3}$ particles/sec. The traces obtained in these tests allow us to simultaneously measure the $(\alpha, p)$ and $(\alpha, n)$ reactions. The measured excitation functions are found to be in good agreement with the published data. Results of these experiments will be published in a separate paper.

\section{Summary}

X-ray bursts can give us information about a wide spectrum of nuclear reactions thought to occur in the atmosphere and the interior of neutron stars. The nuclei involved in these astrophysical scenarios cover isotopes on both sides of the mass valley. The worldwide effort to develop radioactive ion beams during the last 10 years has allowed us to study some of these processes in terrestrial laboratories. For this, intense radioactive beams and detector systems with high efficiency are needed. The active target system described in this contribution has allowed us to make first measurements of fusion and transfer reactions which are of interest to nuclear astrophysics. While the count rates in these first measurements were still limited to about $5 \mathrm{k}$, further improvements such as the addition of fast parallel-plate-avalanche counters before and after MUSIC and the introduction of a gating grid will improve of this limitation by more than an order of magnitude. 


\section{Acknowledgements}

I want to thank my collaborators who were an integral part of the experiments described in this contribution: S. Almaraz, M. Albers, M. Alcorta, M. Avila, P.F. Bertone, P. Carnelli, J. A. Clark, C. Deibel, B. Digiovine, H. Esbensen, J. Fernández Niello, J. M. Figueira, J. Greene, D. Henderson, C. R. Hoffman, C.L. Jiang, B. P. Kay, J. Lai, H. Y. Lee, J. C. Lighthall, S.T. Marley, O. Nusair, T. PalchanHazan, R.C. Pardo, N. Patel, M. Paul, D. Santiago, R. Talwar, C. Ugalde, A. Woodward and A. H. Wuosmaa. This work was supported by the U.S. Department of Energy, Office of Science, Office of Nuclear Physics, under Contract Number DE-AC02-06CH11357 (ANL). This research used resources of ANL's ATLAS facility, which is a DOE Office of Science User Facility.

\section{References}

[1] J. Grindlay et al., Astrophys. J. 205, L127 (1976)

[2] S. E. Woosley and R. T. Taam, Nature 263101 (1976)

[3] http://www.sron.nl/ jeanz/bursterlist.html

[4] W. H. G. Lewin, J. van Paradijs and R. E. Taam, Space Sci. Rev. 62, 223L (1993)

[5] L. Boirin et al., Astron. Astrophys. 465, 559 (2007)

[6] R. Cornelisse et al., Astron. Astrophys. 357, L21 (2000)

[7] H. Schatz and K. E. Rehm, Nucl. Phys. A777, 601 (2006)

[8] http://research;jyu.fi/igisol/JYFLTRAP-masses/ and references therein

[9] B. Harss et al., Rev. Sci. Instrum. 71, 380 (2000)

[10] R. C. Pardo et al., Nucl. Instrum. Methods Phys. Res. Sect. A 790, 1 (2015)

[11] C. M. Deibel et al., Phys. Rev. C 84, 045802 (2011)

[12] J. M. Figueira et al., Nucl. Instrum. Methods Phys. Res. Sect. A 670, 32 (2012)

[13] C. M. Deibel et al., Contribution to the International Conference Nuclei in the Cosmos-11

[14] M. Paul et al., Nucl. Instrum. Methods. Phys. Res. Sect. A 277, 725 (1987)

[15] SMARAGD code, URL: http://nucastro.org/smaragd.html

[16] J. Fisker et al., Astrophys. J 608, 61(2004)

[17] C. J. Horowitz, H. Dussan and D. K. Berry, Phys. Rev. C 77, 045807 (2008)

[18] H. Schatz et al., Nature 505, 62 (2014)

[19] A. S. Umar, V. E. Oberacker and C. J. Horowitz, Phys. Rev. C 85, 055801 (2012)

[20] D. G. Yakovlev, M. Beard, L. R. Gasques and M. Wiescher, Phys. Rev. C 82, 044609 (2010)

[21] W. B. Christie et al., Nucl. Instrum. Methods Phys. Res. Sect. A 255, 466 (1987)

[22] K. Kimura et al., Nucl. Instrum. Methods Phys. Res. Sect. A 297, 190 (1990)

[23] R. N. Boyd et al., Phys. Rev. Lett. 68, 1283 (1992)

[24] Y. Mizoi et al., Nucl. Instrum. Methods. Phys. Res. Sect. A 431, 112 (1999)

[25] P. F. F. Carnelli et al., Nucl. Instrum. Methods Phys. Res. Sect. A 799, 197 (2015)

[26] P. F. F. Carnelli et al., Phys. Rev. Lett. 112, 192701 (2014)

[27] R. A. Dayras, R. G. Stokstad, Z. E. Switkowski and R. M. Wieland, Nucl. Phys. A 266, 153 (1976)

[28] D. G. Kovar et al., Phys. Rev. C 20, 1305 (1979)

[29] M. Avila et al., to be published 